\title{
Tradução e Adaptação Transcultural do Questionário de Pais como Contexto Social
}

\author{
Schwanny Roberta Costa Rambalducci Mofati Vicente ${ }^{1}$, Fabiana Pinheiro Ramos, Kely Maria Pereira de Paula \\ Universidade Federal do Espírito Santo, Vitória-ES, Brasil
}

\section{RESUMO}

Este estudo descreve o processo de adaptação transcultural do Parents as Social Context Questionnaire (PASC), em duas versões (pais e criança), um instrumento que avalia a influência parental no enfrentamento infantil em seis dimensões: afeto, rejeição, estrutura, caos, suporte para autonomia e coerção. A tradução foi realizada por dois especialistas em língua inglesa. Três juízes avaliaram clareza de linguagem, pertinência prática e relevância teórica dos 54 itens ( 24 na versão criança; 30 na versão pais) do instrumento. O coeficiente de validade de conteúdo apresentou, em ambas as versões, classificação substancial $(0,60-0,80)$ ou quase perfeita $(0,80-1,00)$ para todos os itens. O questionário foi aplicado em 60 crianças $(M=9,6$ anos) hospitalizadas e em seus cuidadores $(M=34,9$ anos). As dimensões com maiores médias foram estrutura, autonomia e afeto (protocolo pais); afeto, autonomia e estrutura (protocolo crianças). Nova pesquisa é necessária para dar seguimento à validação do instrumento para a população brasileira.

Palavras-chave: adaptação transcultural, instrumento, crianças, cuidadores, hospitalização.

\section{ABSTRACT - Translation and Cross-Cultural Adaptation of the Parents as Social Context Questionnaire}

This article describes the process of cross-cultural adaptation of the two versions (parents and child) of the Parents as Social Context Questionnaire (PSCQ), an instrument that assesses the parental influence on child coping through six dimensions: warmth, rejection, structure, chaos, autonomy support and coercion. Two specialists in English language performed the translation. Three judges evaluated the language clarity, practical relevance and theoretical relevance of the 54 items ( 24 in the child version, 30 in the parent version) of the instrument. The Content Validity Coefficient presented, in both versions, substantial $(0.60-0.80)$ or almost perfect $(0.80-1.00)$ classifications for all items. The questionnaire was applied with 60 hospitalized children $(M=9.6$ years $)$ and their caregivers $(M=34.9$ years). The dimensions with higher means were structure, autonomy support and warmth (parent version); and warmth, autonomy support and structure (child version). Further research is necessary to continue the validation of the instrument for the Brazilian population.

Keywords: transcultural adaptation; instrument; children; caregivers; hospitalization.

\section{RESUMEN - Traducción y Adaptación Transcultural del Cuestionario de Padres como Contexto Social}

Este estudio describe el proceso de adaptación transcultural del Parents as Social Context Questionnaire (PASC), en dos versiones (padres y niño), un instrumento que evalúa la influencia parental en el enfrentamiento infantil en seis dimensiones: afecto, rechazo, estructura, caos, soporte para autonomía y coerción. La traducción fue realizada por dos expertos en lengua inglesa. Tres jueces evaluaron la claridad del lenguaje, la pertinencia práctica y la relevancia teórica de los 54 ítems (24 en la versión niño; 30 en la versión padres) del instrumento. El Coeficiente de Validez de Contenido presentó, en ambas versiones, clasificación sustancial $(0,60-0,80)$ o casi perfecta $(0,80-1,00)$ para todos los ítems. El cuestionario fue aplicado en 60 niños $(M=9,6$ años $)$ hospitalizados y sus cuidadores $(M=34,9$ años). Las dimensiones con mayores promedios fueron estructura, autonomía y afecto (protocolo padres); afecto, autonomía y estructura (protocolo niño). Una nueva investigación es necesaria para dar seguimiento a la validez del instrumento para la población brasileña.

Palabras clave: adaptación transcultural; instrumento; niños; cuidadores; hospitalización.

Situações estressantes ou adversas são vivenciadas diariamente pelas pessoas e quando avaliadas como ameaçadoras podem repercutir de forma negativa para o indivíduo (Zimmer-Gembeck, Petegem, \& Skinner, 2016). O estresse ocorre quando uma dada situação é percebida como significativa e a demanda excede os recursos que a pessoa possui para lidar com tal evento (Skinner \& Zimmer-Gembeck, 2015). Para manejo dessa situação, o enfrentamento (coping) é definido como ação regulatória, considerando a natureza do evento

${ }^{1}$ Endereço para correspondência: Programa de Pós-Graduação em Psicologia. Prédio Professor Lídio de Souza. Universidade Federal do Espírito Santo. Avenida Fernando Ferrari, 514, 29060-970, Vitória, ES. Tel.: (27) 4009-2501. Fax: (27) 4009-2501. E-mail: schwanny.vicente@gmail.com

Este projeto foi financiado com bolsa de doutorado oferecida pela Coordenação de Aperfeiçoamento de Pessoal de Nível Superior (CAPES). 
estressor. A partir da perspectiva da Teoria Motivacional do Coping - TMC (Motivational Theory of Coping), desenvolvida por Skinner (1999), o processo de enfrentamento envolve a regulação de comportamentos, emoções e orientação motivacional sob estresse (Skinner \& Zimmer-Gembeck, 2015). Em outras palavras, remete à capacidade de autocontrole e ajuste comportamental diante de diversas demandas situacionais (Olson, Sameroff, Lunkenheimer, \& Kerr, 2009).

$\mathrm{Na}$ perspectiva motivacional, o enfrentamento é um processo de esforço ativo e contínuo de reestruturação e reparação de uma ou mais das três necessidades psicológicas básicas do ser humano: competência, autonomia e relacionamento (Ryan \& Deci, 2017). A necessidade de competência diz respeito à interação efetiva com o ambiente na busca de resultados positivos; a autonomia está relacionada com a capacidade de fazer escolhas e a tomada de decisões por conta própria; e a necessidade de relacionamento refere-se à capacidade de ser efetivo nas interações sociais (Ramos, Enumo, \& Paula, 2015). Para lidar com eventos que ameaçam ou desafiam essas três necessidades psicológicas básicas, o indivíduo se utiliza de respostas voluntárias, como planejamento de estratégias e busca de suporte social; e involuntárias (ou automáticas), bebês, por exemplo, possuem várias formas de modular reações de estímulos, com mudança da atenção ou maior proximidade, fazendo com que o enfrentamento nem sempre seja intencional (Skinner, 1999).

São vários os aspectos que influenciam a forma como os indivíduos lidam com os estressores diários, dentre eles pode-se citar o contexto familiar e social, variáveis individuais, como o temperamento, dentre outros (Oliveira et al., 2018; Zimmer-Gembeck et al., 2016). No caso das crianças, o ambiente oferecido pelos pais (ou cuidadores principais) influencia o desenvolvimento do enfrentamento, pois, diante de situações de estresse, estes podem fornecer recursos interpessoais importantes aos filhos, aumentando ou diminuindo pressões durante essas transações (Sameroff, 2009), o que pode se configurar como aspecto positivo ou negativo. Mais especificamente, o contexto social da criança influencia o atendimento de suas necessidades psicológicas básicas, podendo agir como facilitador ou dificultador do enfrentamento de adversidades (Skinner \& Edge, 2002). O processo de enfrentamento também é influenciado pelas transições, mudanças sociais, psicológicas e físicas, que ocorrem ao longo do ciclo vital (Skinner \& Zimmer-Gembeck, 2015). Além disso, crianças, em geral, ao lidar com estressores, adaptam-se ao sistema de enfrentamento interpessoal dos pais (Landers, Friedrich, Jawad, \& Miller, 2016; ZimmerGembeck \& Skinner, 2016).

A TMC, por propor uma perspectiva desenvolvimentista de análise do enfrentamento, sintetiza as principais mudanças que ocorrem ao longo do desenvolvimento, com base em estudos sobre coping de crianças e adolescentes (Skinner \& Zimmer-Gembeck, 2009). As mais importantes no período neonatal estão relacionadas aos sistemas neurológico e fisiológico, sendo o enfrentamento mediado pelos cuidadores. Outra mudança significativa ocorre entre 5 e 7 anos de idade, onde são percebidas alterações na memória, cognição, relações sociais e adoção de um enfrentamento mais intencional. Entre 10 e 12 anos, em função das alterações fisiológicas, há mudanças no padrão de sentimentos e expansão dos relacionamentos sociais e, de 14 a 16 anos, há maior desenvolvimento da autonomia. No final da adolescência, em geral, com as alterações nos papéis sociais e a vivência de experiências desafiadoras, como a inserção na universidade ou mesmo a saída da casa dos pais, o enfrentamento é mais pautado em valores pessoais (Zimmer-Gembeck \& Skinner 2016).

Além de considerar como o nível de desenvolvimento da criança afeta seu enfrentamento, deve-se levar em conta como as práticas parentais influenciam esse processo, tendo em vista que, em grande parte do tempo, a criança encontra-se exposta ao contexto familiar (Crandell, Sandelowski, Leeman, Havill, \& Knafl, 2018; Egeli, 2015). Assim, as interações promotoras de desenvolvimento saudável e que favorecem o processo de enfrentamento incluem o afeto transmitido por pessoas significativas que investem seu tempo, são confiáveis, disponíveis emocionalmente e interessadas no relacionamento com a criança. Estrutura é outra característica importante que abarca aspectos como previsibilidade e consistência do ambiente, fornecendo diretrizes de comportamento e informações claras sobre como atingir resultados desejados. A terceira envolve o incentivo à autonomia, quando há liberdade de expressão e encorajamento para a tomada de decisões (Landers et al., 2016; Skinner, Johnson, \& Snyder, 2005).

Com base nesses pressupostos, Skinner et al. (2005) apresentam seis dimensões importantes para a análise da influência dos comportamentos parentais no enfrentamento do estresse: afeto, rejeição, estrutura, caos, incentivo à autonomia e coerção. Para sua avaliação, os autores desenvolveram o instrumento Parents as Social Context Questionnaire - PASC, cujo objetivo é avaliar o estilo parental, com base nos princípios da TMC. A escala é composta por duas subescalas, uma respondida pelos pais e outra pelos filhos. As subescalas contêm afirmações que contemplam as seis dimensões indicadas, a saber: 1 . afeto, que remete aos sentimentos de amor, carinho e prazer; 2. rejeição, que refere-se a desgosto, aversão, irritabilidade e avaliação negativa; 3. estrutura, envolvendo a provisão de informação visando alcançar metas; 4. caos, quando as orientações são implícitas ou pouco claras sobre como a criança deve agir para obter um resultado; 5. suporte para autonomia, que está relacionado à possibilidade da criança se expressar e a valorização de suas preferências e opiniões; 
e 6. coerção, que se refere a um estilo autocrático, intrusivo, controlador e restritivo.

No estudo de validação do Parents as Social Context Questionnaire (PASC) realizado por Egeli (2015), em uma escala de 30 itens, do tipo Likert com 4 pontos, os pais avaliavam o quanto aquele item estava presente na relação com o filho. A escala considera as seis dimensões propostas por Skinner et al. (2005). A amostra americana foi composta por 404 cuidadores (291 mães e 113 pais) maiores de 18 anos e que tinham uma criança sob seus cuidados, com idade entre 2 e 18 anos. Após análise fatorial, foram retirados três itens da escala. Os resultados indicaram que as seis subescalas tinham consistência interna satisfatória com coeficiente alfa variando entre 0,72 e 0,84 para todos os itens, sendo o alfa total de 0,80 . Desse modo, o instrumento mostrou-se adequado para avaliar as dimensões e a qualidade parental e, ainda, a forma como esse padrão implica nas necessidades psicológicas da criança.

O PASC (relato da criança), composto por 24 itens, também foi traduzido e validado para a população de Singapura, em estudo realizado por Chew e Wang (2013). Após passar por processo de tradução e validação fatorial, o instrumento foi aplicado em 258 atletas com idade entre 13 a 18 anos. Análises fatoriais confirmatórias apresentaram indícios de validade e bons níveis de confiabilidade $(0,85)$.

O estudo realizado por Tolinski (2015), com o mesmo instrumento, investigou engajamento e desempenho acadêmico em crianças do ensino fundamental e verificou as relações com professores e pais. Participaram da pesquisa 354 crianças que estudavam na zona rural do sudoeste de Michigan. Os resultados indicaram que a presença de envolvimento e autonomia no relacionamento pais-filhos e professor-aluno contribuíram para maior engajamento escolar. Assim, os pais, por meio do estabelecimento de estrutura e incentivo à autonomia em casa, influenciam no desenvolvimento do engajamento emocional e comportamental de seus filhos no ambiente escolar, conforme indicado em estudos da área (Egeli, 2015; Skinner et al., 1990; Skinner et al., 2005).

Como os padrões parentais oferecidos às crianças refletem no desfecho de enfrentamento de adversidades, a avaliação adequada do contexto parental é essencial para auxiliar os psicólogos no planejamento de intervenções que visem melhorar o repertório de estratégias da criança, caso não sejam facilitadoras do desenvolvimento. Considerando esse aspecto, o objetivo deste estudo foi realizar a tradução e a adaptação transcultural do Parents as Social Context Questionnaire (PASC) para o português brasileiro, em duas versões, pais/cuidadores e crianças, aplicado em contexto de hospitalização. $\mathrm{O}$ processo de tradução e adaptação da escala integra uma pesquisa mais ampla sobre o enfrentamento da hospitalização em crianças.

\section{Método}

\section{Participantes}

Neste estudo, participaram quatro profissionais formados em línguas/inglês, com mais de cinco anos de conclusão do curso e com experiência na área. Também participaram do estudo três psicólogos, com nível de mestrado, estudiosos do desenvolvimento infantil e com conhecimento sobre a TMC.

Após a tradução do PASC e avaliação dos juízes, o instrumento foi aplicado em 60 crianças internadas em diversas enfermarias de um hospital estadual infantil do estado do Espírito Santo, entre 7 e 12 anos, com idade média de 9,6 anos $(D P=1,7)$, devidamente acompanhadas por um cuidador no período da coleta de dados, realizada entre julho e novembro de 2016. A escolha desse contexto se relaciona à presença de diversos estressores inerentes a uma internação infantil por doença ou tratamento, requerendo envolvimento parental constante (Oliveira et al., 2018). Todas as crianças possuíam capacidade física para participar, sem comprometimento neurológico que limitasse o preenchimento dos instrumentos. Além desses critérios, foram excluídas da amostra crianças que não possuíam a concordância de um dos responsáveis legais ou que indicaram ao pesquisador que não desejavam participar do estudo. Os cuidadores deveriam estar na condição de acompanhante da criança durante todo o processo de hospitalização, residir na mesma casa, e ter um papel importante na sua educação.

O tempo médio de internação foi de 3,9 dias até o início da coleta dos dados e as crianças estavam internadas nas seguintes enfermarias: cirúrgica $(N=29)$, ortopedia $(N=20)$, enfermaria geral $(N=9)$ e infectologia $(N=2)$. Na amostra de crianças $(N=60), 33$ eram meninos. As crianças foram hospitalizadas para a realização de procedimentos cirúrgicos $(N=28)$, tratamento de alguma doença $(N=21)$, em função de algum acidente, como atropelamento ou fratura $(N=8)$, ou para investigação médica, por exemplo, febre ou dor sem motivo aparente $(N=3)$. Quanto ao nível de escolaridade, as crianças estavam no $1^{\circ}$ ano $(N=7), 2^{\circ}$ ano $(N=8), 3^{\circ}$ ano $(N=12)$, $4^{\circ}$ ano $(N=7), 5^{\circ}$ ano $(N=13), 6^{\circ}$ ano $(N=11)$ e $7^{\circ}$ ano $(N=2)$. Cabe ressaltar que todas estavam alfabetizadas no período da data da coleta de dados. A maioria era residente no estado do Espírito Santo $(N=55)$, sendo 31 da Grande Vitória e 24 do interior. Duas crianças residiam no interior da Bahia e três crianças moravam no interior do estado de Minas Gerais.

A maioria das crianças estava acompanhada pela mãe $(N=45)$, seguida pelo pai $(N=9)$ e irmão/irmã $(N=2)$, tio/ tia $(N=2)$ e avô/avó $(N=2)$. A média de idade dos cuidadores foi de 34,9 anos. Quanto ao nível de escolaridade, a maioria cursou ensino médio completo $(N=22)$, seguido por fundamental incompleto $(N=13)$, médio incompleto $(N=9)$, fundamental completo $(N=8)$, superior incompleto $(N=6)$, ensino superior $(N=1)$ e iletrado $(N=1)$. 
Em relação ao número de membros da família, a média foi de 4,53 pessoas. Entre os cuidadores, a maioria possuía alguma ocupação $(N=32)$. Grande parte contava com ajuda durante o período de internação da criança $(N=47)$, na maioria das vezes, fornecida somente pela família $(N=35)$, seguida de ajuda exclusiva de amigos $(N=6)$. Alguns cuidadores contavam com auxílio de ambos $(N=4)$ e um cuidador disse receber ajuda da família, equipe e governo. Os outros relataram não receber qualquer tipo de ajuda $(N=13)$. No que tange à crença religiosa, a maioria dos familiares relatou pertencer a alguma igreja $(N=49)$.

\section{Instrumentos}

$\mathrm{Na}$ etapa de tradução, as duas versões da escala (cuidador e criança), com conteúdo original foram enviadas aos profissionais de línguas. Para a tradução reversa, a escala na versão em português foi utilizada. Os profissionais de Psicologia utilizaram uma planilha com os itens da escala para análise dos critérios de clareza de linguagem, pertinência prática, relevância teórica e dimensão avaliada. Cada item devia ser analisado a partir de um roteiro descritivo conceituando esses critérios e cada uma das dimensões do instrumento (Skinner et al., 2005). Para a coleta de dados foi utilizado um Protocolo de Identificação, respondido pelo cuidador, que teve como objetivo levantar dados gerais da amostra. Em seguida, o cuidador respondeu o QPCS (versão pais) e, em momento separado, foi aplicado o QPCS com as crianças.

\section{Procedimentos de Coleta de Dados}

A tradução transcultural de uma escala requer o cumprimento de determinadas etapas (Borsa, Damásio, \& Bandeira, 2012). Após a tradução e adaptação do instrumento pelos dois profissionais especialistas na língua inglesa, a pesquisadora principal deste estudo fez a síntese das versões. Na sequência, outros três juízes especialistas na área avaliada pelo instrumento realizaram a avaliação da versão traduzida considerando os aspectos citados: clareza de linguagem em cada um dos itens; pertinência prática, considerando se o item investiga o conceito de interesse na população estudada; e o nível de associação entre o item e a teoria estudada. Por fim, os profissionais precisavam estimar a adequação do item em relação à dimensão avaliada, considerando a teoria que subsidia a análise (Cassepp-Borges, Balbinotti, \& Teodoro, 2010). Após a avaliação de cada item, considerando esses quatro aspectos, a versão foi enviada aos dois especialistas em línguas para a tradução reversa.

Após a tradução e adaptação do Parents as Social Context Questionnaire - PASC passou a ser denominado de Questionário de Pais como Contexto Social - QPCS. A versão para crianças é composta por 24 sentenças (ex.: "Meus pais me dizem que me amam."), enquanto que o relato dos pais é formado por 30 itens (ex.: "Digo ao meu filho que o amo."). Cada um deve responder ao protocolo separadamente. Ambas as versões apresentam uma escala do tipo Likert de quatro pontos, indicando o quanto a sentença é verdadeira (sendo 1 "não é nada verdadeiro"; 2 "é pouco verdadeiro"; 3 "muito verdadeiro"; e 4 "extremamente verdadeiro").

Ao final de todas as etapas descritas acima, o projeto completo, ao qual este estudo faz parte, foi submetido ao Comitê de Ética da Universidade Federal do Espírito Santo e aprovado com parecer número 1.579.291, conforme resolução 510 do Conselho Nacional de Saúde (Brasil, 2012). Com a autorização da instituição, a pesquisa foi iniciada, mediante concordância tanto do cuidador quanto da criança, que assinaram os Termos de Consentimento Livre e Esclarecido e de Assentimento.

\section{Análise dos Dados}

Para a análise de tradução da escala, baseado nas respostas dos juízes, foi realizado o coeficiente de validade de coeficiente (CVC), que mede a concordância entre os juízes, e utilizado o kappa, para reduzir deficiências de métodos estatísticos, aplicado em análises com variáveis categóricas. Para o cálculo de CVC total, foi realizado o cálculo de CVC inicial $\left(C V C_{i}=M_{x} / V_{\max x}\right.$, onde $M_{x}$ é a média das notas de cada item e $V_{\text {máx }}$ refere-se ao valor máximo que cada item pode receber. Para avaliação de cada item os juízes pontuavam considerando uma escala Likert de 1 a 5 pontos, logo, o valor máximo era 5), em seguida foi realizado o cálculo de erro $\left(P e_{i}\right)\left(P e_{i}=(1 / J)^{J}\right.$, onde $J$ é a quantidade de juízes), que é o mesmo para as modalidades clareza de linguagem, pertinência prática e relevância teórica. Em seguida foi calculado o CVC final $\left(C V C_{c}=C V C_{i}-P e_{i}\right.$, onde). O CVC total é a média do CVC final de cada item para cada modalidade (Cassepp-Borges, Balbinotti, \& Teodoro, 2010).

O kappa é o cálculo para verificação da intensidade de concordância de dois ou mais juízes para classificação de itens. O índice de significância do kappa é realizado para verificar se a concordância é razoável, onde um resultado de kappa $=1$ indica nível máximo de concordância e 0 indica que o grau de concordância não existe, e um resultado negativo não pode ser interpretado.

Como critério de interpretação dos resultados dos cálculos estatísticos, os seguintes valores foram utilizados: discordância $(<0)$, quase nenhum $(0-0,20)$; pequeno $(0,20-0,40)$; moderado $(0,40-0,60)$; substancial $(0,60-$ $0,80)$; e quase perfeito $(0,80-1)$. Quanto mais próximo de 1 mais fidedigno é o item. Para este trabalho, considerou-se a necessidade de utilizar apenas itens com CVC dos grupos substancial e quase perfeito, conforme recomendado na literatura (Cassepp-Borges et al., 2010; Egeli, 2015; Laboratório de Epidemiologia e Estatística, 2017).

Os dados foram organizados em planilhas e, posteriormente, foi realizada análise estatística. Para o cálculo do CVC, foi utilizado o software Excel 2016, e para o cálculo do kappa foi utilizada a ferramenta on-line disponível pelo Laboratório de Epidemiologia e Estatística (2017). 


\section{Resultados}

\section{Análise do Processo de Tradução e Adaptação do Instrumento}

Antes do início da coleta de dados, foi realizado o cálculo de coeficiente de validade do conteúdo (CVC) para cada item da escala para as modalidades clareza de linguagem (CL), pertinência prática $(\mathrm{PP})$ e relevância teórica (RL) (Cassepp-Borges et al., 2010), em ambas as versões do instrumento (pais e criança). Para avaliar a concordância entre os juízes em relação às dimensões da escala, foi calculado o coeficiente kappa (Borsa et al., 2012).

$\mathrm{Na}$ escala dos pais, todos os itens estavam na categoria de concordância substancial, considerando o resultado do CVC, para os critérios clareza de linguagem
(CL), pertinência prática $(\mathrm{PP})$ e relevância teórica $(\mathrm{RT})$, conforme cálculo descrito na metodologia do estudo. Os itens do instrumento com menor valor do cálculo de validade de conteúdo (CVC) foram: o dois $(\mathrm{CVC}=0,63)$, o três $(\mathrm{CVC}=0,63)$ e o trinta $(\mathrm{CVC}=0,63)$, todos para o critério clareza de linguagem. $\mathrm{O}$ item três $(\mathrm{CVC}=0,63)$ também teve CVC baixo para pertinência prática. E o vinte e sete $(\mathrm{CVC}=0,63)$ teve o mesmo $\mathrm{CVC}$ para relevância teórica. No critério clareza de linguagem do total de 30 itens, 17 obtiveram CVC quase perfeito $(0,80-1)$, o CVC total para o critério nesta escala foi de 0,79. Em pertinência prática, 16 itens estavam com CVC quase perfeito, sendo o CVC total também 0,79. Já, no critério relevância teórica, 12 itens foram classificados como quase perfeito e o CVC total o foi mesmo de clareza de linguagem e pertinência prática $(0,79)$ (Tabela 1$)$.

Tabela 1

Cálculo de Coeficiente de Validade do Conteúdo para Cada Item da Escala dos Pais para Clareza de Linguagem, Pertinência Prática e Relevância Teórica

\begin{tabular}{|c|c|c|c|}
\hline \multirow{2}{*}{ Item } & \multicolumn{3}{|c|}{ CVC } \\
\hline & CL & $\mathrm{PP}$ & RT \\
\hline 1. Eu sei muito sobre o que se passa com o meu filho. & 0,70 & 0,83 & 0,70 \\
\hline 2. Eu sei exatamente como meu filho se sente. & 0,63 & 0,76 & 0,76 \\
\hline 3. Eu faço coisas especiais com meu filho. & 0,63 & 0,63 & 0,70 \\
\hline 4. Eu separo um tempo para conversar com meu filho sobre o que é importante para ele. & 0,83 & 0,83 & 0,76 \\
\hline 5. Permito que meu filho saiba que eu o amo. & 0,76 & 0,83 & 0,83 \\
\hline 6. Eu não entendo meu filho muito bem. & 0,90 & 0,70 & 0,70 \\
\hline 7. Às vezes é difícil gostar do meu filho. & 0,83 & 0,83 & 0,90 \\
\hline 8. Por vezes, as exigências que meu filho faz são um fardo, são pesadas. & 0,70 & 0,83 & 0,83 \\
\hline 9. Meu filho precisa de mais tempo do que eu tenho para dar a ele. & 0,83 & 0,83 & 0,83 \\
\hline 10. Às vezes sinto que não posso estar ao lado do meu filho quando ele precisa de mim. & 0,83 & 0,76 & 0,76 \\
\hline 11. Eu deixo claro o que irá acontecer se meu filho não seguir nossas regras. & 0,76 & 0,76 & 0,83 \\
\hline 12. Eu deixo claro ao meu filho sobre o que eu espero dele. & 0,90 & 0,83 & 0,90 \\
\hline 13. Eu quero que o meu filho siga as regras da nossa família. & 0,70 & 0,76 & 0,76 \\
\hline 14. Quando eu digo ao meu filho que irei fazer algo, eu faço. & 0,83 & 0,83 & 0,83 \\
\hline 15. Se meu filho tem um problema, eu o ajudo a descobrir o que fazer. & 0,90 & 0,90 & 0,83 \\
\hline 16. Eu deixo meu filho sair impune de coisas que eu realmente não deveria permitir. & 0,76 & 0,76 & 0,76 \\
\hline 17. Quando meu filho se mete em encrenca, minha reação não é muito previsível. & 0,83 & 0,70 & 0,76 \\
\hline 18. Meu filho não parece saber o que eu espero dele. & 0,83 & 0,83 & 0,76 \\
\hline 19. Eu mudo muito as regras da casa. & 0,83 & 0,83 & 0,83 \\
\hline 20. Eu posso ficar bravo com meu filho do nada. & 0,76 & 0,70 & 0,76 \\
\hline 21. Eu encorajo meu filho a expressar seus sentimentos mesmo quando são difíceis de se escutar. & 0,90 & 0,90 & 0,90 \\
\hline 22. Eu encorajo meu filho a expressar suas opiniões mesmo quando não concordo com elas. & 0,90 & 0,90 & 0,90 \\
\hline 23. Eu confio no meu filho. & 0,90 & 0,83 & 0,76 \\
\hline 24. Eu encorajo meu filho a ser verdadeiro consigo mesmo. & 0,90 & 0,90 & 0,90 \\
\hline 25. Eu espero que meu filho diga o que ele realmente pensa. & 0,83 & 0,83 & 0,83 \\
\hline 26. Meu filho discute comigo a cada oportunidade. & 0,70 & 0,70 & 0,76 \\
\hline 27. Para conseguir com que meu filho faça algo, tenho que gritar com ele. & 0,90 & 0,76 & 0,63 \\
\hline 28. Há muitas coisas que eu não posso permitir que meu filho decida por conta própria. & 0,70 & 0,70 & 0,70 \\
\hline 29. Às vezes sinto que tenho que pressionar meu filho para que faça as coisas. & 0,70 & 0,76 & 0,76 \\
\hline 30. Estou em constante disputa de poder com meu filho. & 0,63 & 0,76 & 0,70 \\
\hline
\end{tabular}


Para o cálculo da concordância entre as dimensões, foi calculado o kappa, conforme descrito no método. Todas as dimensões obtiveram o kappa médio substancial, sendo o menor na dimensão incentivo à autonomia $(k=0,662)$. O maior índice obtido foi na dimensão estrutura $(k=0,855)$, sendo classificado como quase perfeito. O valor do kappa geral também foi substancial $(k=0,786)$ $(p<0,001)$.

Para o cálculo da escala das crianças, o CVC total está na categoria substancial, assim como a escala dos pais. Em clareza de linguagem, 18 itens obtiveram CVC maior que 0,8 , estando assim na categoria quase perfeito. Dentre os itens do instrumento de menor valor na análise estão o dezesseis e o vinte e dois $(\mathrm{CVC}=0,70)$. Para pertinência prática, 23 itens estão na categoria quase perfeito, somente o item vinte e dois $(\mathrm{CVC}=0,73)$ está na categoria substancial. Em relevância teórica a maioria dos itens pertence à categoria quase perfeito, sendo somente os itens dez e vinte e dois na categoria substancial $(\mathrm{CVC}=0,73)$ (Tabela 2).

Tabela 2

Cálculo de Coeficiente de Validade do Conteúdo para Cada Item da Escala das Crianças para Clareza de Linguagem, Pertinência Prática e Relevância Teórica

\begin{tabular}{lccc}
\hline \multicolumn{1}{c}{ Item } & CVC \\
\cline { 2 - 4 } & CL & PP & RT \\
\hline 1. Meus pais deixam que eu saiba que eles me amam. & 0,76 & 0,83 & 0,83 \\
2. Meus pais gostam de ficar comigo. & 0,90 & 0,87 & 0,93 \\
3. Meus pais estão sempre contentes ao me ver. & 0,83 & 0,80 & 0,87 \\
4. Meus pais me acham especial. & 0,83 & 0,87 & 0,87 \\
5. Às vezes me pergunto se meus pais gostam de mim. & 0,83 & 0,87 & 0,80 \\
6. Meus pais acham que estou sempre atrapalhando. & 0,76 & 0,87 & 0,87 \\
7. Meus pais me fazem sentir como se eu não fosse desejado por eles. & 0,90 & 0,87 & 0,87 \\
8. Nada que eu faça é bom o bastante para meus pais. & 0,83 & 0,87 & 0,87 \\
9. Quando eu quero fazer algo, meus pais me mostram como fazer. & 0,83 & 0,80 & 0,80 \\
10. Quando quero compreender como algo funciona, meus pais me explicam. & 0,83 & 0,87 & 0,73 \\
11. Caso eu tenha algum problema, meus pais me ajudam a descobrir o que fazer. & 0,83 & 0,87 & 0,87 \\
12. Meus pais me explicam os motivos para as regras da nossa família. & 0,83 & 0,87 & 0,87 \\
13. Quando os meus pais prometem algo, eu não sei se eles irão cumprir. & 0,90 & 0,87 & 0,87 \\
14. Quando meus pais dizem que irão fazer algo, às vezes eles não fazem aquilo que disseram. & 0,76 & 0,87 & 0,87 \\
15. Meus pais sempre mudam as regras comigo. & 0,76 & 0,80 & 0,87 \\
16. Meus pais ficam nervosos comigo do nada. & 0,70 & 0,80 & 0,87 \\
17. Meus pais confiam em mim. & 0,83 & 0,80 & 0,80 \\
18. Meus pais me aceitam do jeito que sou. & 0,83 & 0,87 & 0,87 \\
19. Meus pais permitem que eu faça coisas que eu considero importante. & 0,90 & 0,93 & 0,93 \\
20. Meus pais tentam entender meu ponto de vista. & 0,83 & 0,87 & 0,80 \\
21. Meus pais estão sempre me dizendo o que fazer. & 0,83 & 0,87 & 0,87 \\
22. Meus pais mandam em mim. & 0,70 & 0,73 & 0,73 \\
23. Meus pais acham que há somente um jeito certo de fazer as coisas - o jeito deles. & 0,90 & 0,87 & 0,87 \\
24. Meus pais dizem "não" a tudo. & 0,83 & 0,87 & 0,93 \\
\hline
\end{tabular}

O cálculo do kappa também foi realizado para o questionário das crianças, de modo a verificar a concordância entre as dimensões. Todas as dimensões obtiveram o kappa médio substancial, sendo as dimensões com menores índices caos e incentivo à autonomia $(k=0,6)$. A dimensão coerção obteve maior índice de concordância, com classificação quase prefeita $(k=0,906)$. O valor do kappa geral também foi substancial $(k=0,731)(p<0,001)$.

\section{Aplicação do Instrumento no Contexto de Hospitalização}

Para análise do relato dos pais e das crianças, foi realizada a média do escore para cada dimensão avaliada. Em relação às respostas dos pais, a dimensão de maior média foi estrutura $(M=17,75)$, seguida de incentivo à autonomia $(M=17,71)$ e afeto $(M=17,48)$; e as dimensões de menor média foram as negativas, rejeição $(M=9,32)$, 
caos $(M=9,51)$ e coerção $(M=12,78)$. Os resultados das crianças foram similares, com maior média em dimensões positiva e menores médias em dimensões negativas. Afeto $(M=15,08)$ foi a dimensão de maior média para as crianças, seguido de incentivo à autonomia $(M=14,16) \mathrm{e}$ estrutura $(M=14,00)$; as dimensões de menores médias para este grupo foram rejeição $(M=7,83)$, caos $(M=8,76)$ e coerção $(M=12,10)$.

Tabela 3

Comparação entre as Médias do Questionário de Pais como Contexto Social Relato de Pais e Crianças

\begin{tabular}{|c|c|c|c|c|c|c|c|c|}
\hline & Dimensão & Média & F1 & $\mathrm{F} 2$ & F3 & $\mathrm{F} 4$ & F5 & F6 \\
\hline Cuidador & \multirow{2}{*}{ Afeto } & 17,48 & 25 & 13 & 19 & 0 & 1 & 2 \\
\hline Criança & & 15,08 & 45 & 9 & 2 & 4 & 0 & 0 \\
\hline \multirow{2}{*}{$\begin{array}{l}\text { Cuidador } \\
\text { Criança }\end{array}$} & \multirow{2}{*}{ Rejeição } & 9,32 & 0 & 1 & 2 & 9 & 28 & 20 \\
\hline & & 7,83 & 0 & 1 & 2 & 7 & 22 & 28 \\
\hline \multirow{2}{*}{$\begin{array}{l}\text { Cuidador } \\
\text { Criança }\end{array}$} & \multirow{2}{*}{ Estrutura } & 17,75 & 26 & 15 & 15 & 3 & 1 & 0 \\
\hline & & 14,00 & 28 & 11 & 14 & 3 & 4 & 0 \\
\hline \multirow{2}{*}{$\begin{array}{l}\text { Cuidador } \\
\text { Criança }\end{array}$} & \multirow[b]{2}{*}{ Caos } & 9,51 & 2 & 1 & 1 & 16 & 19 & 21 \\
\hline & & 8,76 & 0 & 0 & 4 & 13 & 28 & 15 \\
\hline \multirow{2}{*}{$\begin{array}{l}\text { Cuidador } \\
\text { Criança }\end{array}$} & \multirow{2}{*}{ Incentivo à autonomia } & 17,71 & 32 & 12 & 8 & 5 & 3 & 0 \\
\hline & & 14,16 & 31 & 10 & 12 & 5 & 0 & 2 \\
\hline \multirow{2}{*}{$\begin{array}{l}\text { Cuidador } \\
\text { Criança }\end{array}$} & \multirow{2}{*}{ Coerção } & 12,78 & 10 & 3 & 7 & 24 & 13 & 3 \\
\hline & & 12,10 & 8 & 4 & 13 & 27 & 7 & 1 \\
\hline
\end{tabular}

Nota. F1=dimensão mais frequentemente utilizada; F2=segunda dimensão mais frequentemente utilizada; F3=terceira dimensão mais frequentemente utilizada; F4=terceira dimensão mais frequentemente não utilizada; F5=segunda dimensão mais frequentemente não utilizada; e F6=dimensão mais frequentemente não utilizada

Na percepção da maioria dos cuidadores, a dimensão mais oferecida por eles aos seus filhos foi incentivo à autonomia $(N=32)$, seguido por estrutura $(N=26)$ e afeto $(N=25)$, enquanto as menos utilizadas pela maior parte dos cuidadores foram caos $(N=21)$ e rejeição $(N=20)$. Para as crianças, as dimensões mais presentes na relação com os pais foram afeto $(N=45)$, incentivo à autonomia $(N=31)$ e estrutura $(N=28)$. As dimensões menos presentes nas relações com os pais, na opinião das crianças foram rejeição $(N=28)$ e caos $(N=15)$ (Tabela 3). Este resultado mostra que a dimensão negativa mais presente na relação cuidador-criança na percepção de ambos os grupos é coerção, sendo a terceira menos utilizada na opinião de crianças $(N=27)$ e cuidadores $(N=24)$, sendo que um número maior de crianças percebe a presença mais frequente dessa dimensão.

\section{Discussão}

O presente estudo teve como objetivo realizar a tradução e a adaptação transcultural do Questionário de Pais como Contexto Social - QPCS (versão pais e crianças), com o intuito de investigar o contexto parental dos pais de crianças hospitalizadas. Inicialmente, após realização das etapas de tradução, foi feito o cálculo de coeficiente de validade do conteúdo para cada item da escala, analisando a clareza de linguagem, pertinência prática e relevância teórica, além de ter sido calculado o coeficiente kappa para avaliar a concordância dos juízes sobre as dimensões da escala (Borsa et al., 2012). Para todos os itens, foram obtidos valores substanciais ou quase perfeitos, considerados por Cassepp-Borges et al. (2010) como adequados para validade do conteúdo e utilização do questionário. Mesmo os itens que apresentaram valores mais baixos estão dentro do critério indicado pela literatura e adotado nesta pesquisa, e ainda, a maioria dos itens dos dois questionários (versão pais e versão crianças) foram classificados como quase perfeitos, considerando os resultados decorrentes análise estatística, indicando sua adequação.

A escala foi aplicada em crianças e em seus cuidadores, entretanto, a amostra de cuidadores foi majoritariamente composta por mães. Tal fato ocorreu porque, na maior parte das vezes, são elas que acompanham seus filhos durante o processo de hospitalização. Apesar de a escala ser destinada a cuidadores de forma geral, incluindo pais, mães e responsáveis, essa é uma limitação da presente pesquisa. Espera-se que pesquisas futuras possam 
utilizar a escala com outros tipos de cuidadores e em outros contextos. O modelo da TMC, com as seis dimensões de estilos parentais (Skinner et al., 2005), obteve coeficiente adequado nos estudos de validação (Chew \& Wang, 2013; Egeli, 2015) e mostrou-se adequado também em estudo de avaliação do contexto parental (Tolinski, 2015). Uma vez que os estilos parentais possuem papel importante ao longo do desenvolvimento da criança (Álvarez-García et al., 2016; Power, 2013), ferramentas de avaliação, como o QPCS, podem ser importantes no planejamento de intervenções, considerando as particularidades das crianças e de seus cuidadores (Crandell et al., 2018). Além disso, sabe-se da importância do envolvimento dos pais na adesão de tratamentos de saúde, agindo como facilitadores do desenvolvimento e respeitando a necessidade de autonomia da criança (Landers et al., 2016). No que diz respeito à caracterização da amostra, a maior parte das crianças participantes estava internada na enfermaria cirúrgica, onde o tempo de internação é menor e onde ocorre maior rotatividade devido aos procedimentos realizados. O tempo médio de hospitalização até o primeiro dia de coleta foi de 3,97 dias. Sabe-se que o contexto de hospitalização interfere na rotina, alterando o estado emocional da criança, gerando medo e apreensão (Hostert et al., 2014). Além disso, os efeitos podem ser observados em longo prazo, incluindo o retorno da criança para as atividades cotidianas após a hospitalização (Oliveira et al., 2018).

O ambiente hospitalar envolve, assim, a participação ativa de cuidadores (Duarte, Zanini, \& Nedel, 2012) e pode repercutir de forma importante também no seu bem-estar emocional (Foster, Young, Mitchell, Van, \& Curtis, 2017). Nesse contexto, os pais precisam manter-se focados no tratamento e na saúde emocional das crianças, pois serão suporte para seus filhos ao longo de todo o tratamento (Scholten et al., 2015), oferecendo (ou não) contextos mais adequados para o desenvolvimento.

A aplicação do questionário mostrou que os pais oferecem um contexto em que se fazem presentes diversas dimensões, incluído as facilitadoras e não facilitadoras do enfrentamento da hospitalização por parte da criança. As implicações do uso de estratégias agrupadas nas dimensões negativas do instrumento se referem a proporcionar à criança experiência de ameaça, desencadear tendências de ações destrutivas, afastar recursos pessoais de regulação e levar a uma relação inadequada da emoção e do comportamento (Zimmer-Gembeck \& Skinner, 2016). Nesse sentido, os efeitos negativos do contexto parental da criança no desenvolvimento do enfrentamento podem ser devastadores, levando a processos de adaptação bastante falhos. Ressalta-se a importância dos pais no desenvolvimento da criança e, ainda, a necessidade de um olhar atento dos profissionais presentes no ambiente hospitalar, a fim de promover intervenções direcionadas à capacitação dos cuidadores para estimulação adequada da criança, visando diminuir as dimensões negativas nas interações e aumentar ainda mais contextos afetivos estruturados, incentivando a autonomia das crianças.

Os estudos sobre esses efeitos do contexto parental em ambiente hospitalar ainda são escassos e, por isso, pouco conclusivos, mas sabe-se que fatores como afetividade, estrutura e incentivo à autonomia são importantes para o desenvolvimento infantil adequado (ÁlvarezGarcía et al., 2016; Skinner et al., 2005). Novos estudos precisam ser realizados para que se identifique se essas dimensões estão diretamente relacionadas com o enfrentamento da hospitalização e com a adesão ao tratamento. Os novos estudos devem incluir, ainda, relatos de pais, crianças e equipe de saúde. Espera-se que futuramente sejam realizados estudos de validação do QPCS, apresentando parâmetros psicométricos para a população brasileira, de modo semelhante ao que foi realizado para crianças americanas (Egeli, 2015) e adolescentes de Singapura (Chew \& Wang, 2015).

Considerando que o relacionamento entre pais e filhos pode afetar o repertório de estratégias de enfrentamento desenvolvidas e utilizadas pelas crianças em situação de estresse, a avaliação adequada de tais padrões pode viabilizar intervenções mais direcionadas a pais e cuidadores, diminuindo o risco e a vulnerabilidade de crianças em contextos estressantes, como é o caso da hospitalização. Em um ambiente com presença de cuidadores que apresentam um contexto de fornecimento de afeto, estrutura e suporte para a autonomia, as crianças desenvolvem repertório de estratégias de enfrentamento mais apropriado, minimizando o impacto da hospitalização no seu desenvolvimento e favorecendo-o.

\section{Referências}

Álvarez-García, D., García, T., Barreiro-Collazo, A., Dobarro, A., \& Antúnez, Á. (2016). Parenting style dimensions as predictors of adolescent antisocial behavior. Frontiers in Psychology, 7(2016), 1-9. doi: 10.3389/fpsyg.2016.01383

Borsa, J. C., Damásio, B. F., \& Bandeira, D. R. (2012). Adaptação e validação de instrumentos psicológicos entre culturas: Algumas considerações. Paideia, 22(53), 423-432. doi: 10.1590/1982-43272253201314

Cassepp-Borges, V., Balbinotti, M. A. A., \& Teodoro, M. L. M. (2010). Tradução e validação de conteúdo: Uma proposta para a adaptação de instrumentos. Em Luiz Pasquali (Ed.), Instrumentação Psicológica: Fundamentos e práticas (pp. 506-520). Porto Alegre: Artmed. 
Chew, E. W. C., \& Wang, J. C. K. (2013). Parents as social context in youth sport: A validation of the PASCQ with adolescent Singapore athletes. International Sports Studies, 35(2), 34-46. Recuperado de http://hdl.handle.net/10497/17029

Crandell, J. L., Sandelowski, M., Leeman, J., Havill, N. L., \& Knafl, K. (2018). Parenting behaviors and the well-being of children with a chronic physical condition. Families, Systems, E Health, 36(1), 45-61. doi: 10.1037/fsh0000305

Duarte, M. de L. C., Zanini, L. N., \& Nedel, M. N. B. (2012). O cotidiano dos pais de crianças com câncer e hospitalizadas. Revista Gaúcha de Enfermagem, 33(3), 111-118. doi: 10.1590/S1983-14472012000300015

Egeli, N. A. (2015). Measuring and Predicting Parenting Style Using Self-Determination Theory. (Thesis submitted of requirement of the degree of doctor of Philosophy in Counselling Psychology). Department of Educational Psychology of University of Alberta, Edmonton, Canadá. doi: 10.1017/CBO9781107415324.004

Foster, K., Young, A., Mitchell, R., Van, C., \& Curtis, K. (2017). Experiences and needs of parents of critically injured children during the acute hospital phase: A qualitative investigation. Injury, 48(1), 114-120. doi: 10.1016/j.injury.2016.09.034

Hostert, P. C. da C. P., Enumo, S. R. F., \& Loss, A. B. M. (2014). Brincar e problemas de comportamento de crianças com câncer de classes hospitalares. Psicologia: Teoria e Prática, 16(1), 127-140. Recuperado de http://pepsic.bvsalud.org/pdf/ptp/v16n1/11.pdf

Laboratório de Epidemiologia e Estatística (2017). Recuperado de http://www.lee.dante.br/pesquisa/kappa/

Landers, S. E., Friedrich, E. A., Jawad, A. F., \& Miller, V. A. (2016). Examining the interaction of parental involvement and parenting style in predicting adherence in youth with type 1 diabetes. Families, systems \& health: The journal of collaborative family healthcare, 34(1), 41-50. doi: $10.1037 /$ fsh0000183

Brasil. Ministério da Saúde. (2012). Resolução No 466, de 12 de dezembro de 2012, Pub. L. No. 466/12, 59. Brasil. Recuperado de http:// conselho.saude.gov.br/resolucoes/2012/Reso466.pdf

Oliveira, C. M. M., Amorim, J. C., Alves, I. A., Dias, T. L., Silveira, K. A., \& Enumo, S. R. F. (2018). Estresse, autorregulação e risco psicossocial em crianças hospitalizadas. Saúde e Desenvolvimento Humano, 6(1), 39-48. doi: 10.18316/sdh.v6i1.4132

Olson, S. I., Sameroff, A., Lunkenheimer, E. S., \& Kerr, D. (2009). Self-regulatory processes in the development of disruptive behavior problems: The preschool to school transition. Em S. L. Olson \& A. J. Sameroff (Eds.), Biopsychosocial regulatory processes in the development of childhood behavioral problems (pp. 144-185). New York: Cambridge University Press.

Power, T. G. (2013). Parenting Dimensions and Styles: A brief history and recommendations for future research. Childhood Obesity, 9(s1), S-14-S-21. doi: 10.1089/chi.2013.0034

Ramos, F. P., Enumo, S. R. F., \& Paula, K. M. P. de. (2015). Teoria Motivacional do Coping: uma proposta desenvolvimentista de análise do enfrentamento do estresse. Estudos de Psicologia (Campinas), 32(2), 269-280. doi: 10.1590/0103-166X2015000200011

Ryan, R. M., \& Deci, E. L. (2017). Self-Determination Theory: Basic Psychological Needs in Motivation, Development and Wellness. New York: Guilford Press.

Sameroff, A. (2009). The transactional model. Em A. Sameroff (Ed.), The transactional model of development: How children and contexts shape each other (pp. 3-21). Washington, DC, US: American Psychological Association. doi: 10.1037/11877-001

Scholten, L., Willemen, A. M., Napoleone, E., Maurice-Stam, H., Last, B. F., Dijk-Lokkart, E. M. van, Zandbelt, N., Ensink, E., Grootenhuins, M. A., Schuengel, C. (2015). Moderators of the Efficacy of a Psychosocial Group Intervention for Children With Chronic Illness and Their Parents: What Works for Whom? Journal of Pediatric Psychology, 40(2), 214-227. doi: 10.1093/jpepsy/jsu092

Skinner, E. A. (1999). Action regulation, coping, and development. Em J. Brandtstandter \& R. M. Lerner (Eds.), Action and self-development: Theory and research through the lifespan (pp. 465-503). Thousand Oaks, CA, US: Sage Publications, Inc .doi: 10.4135/9781452204802.n16

Skinner, E. A., \& Edge, K. (2002). Parenting, motivation, and development of coping. Em L. J. Crockett (Ed.), Nebraska Symposium on Motivation: Motivation, agency, and the life course (pp. 77-143). Lincoln NB: University of Nebraska Press.

Skinner, E. A., Johnson, S., \& Snyder, T. (2005). Six Dimensions of Parenting: A Motivational Model. Parenting: Science and Practice, 5(2), 175-235. doi: 10.1207/s15327922par0502_3

Skinner, E. A., \& Zimmer-Gembeck, M. J. (2009). Challenges to the developmental study of coping. Em E. A. Skinner \& M. J. ZimmerGembeck (Eds.), Coping and the developmentof regulation: New directions for child and adolescent development (pp. 5-17). San Francisco: JosseyBass. doi: 10.1002/cd.239

Skinner, E. A., \& Zimmer-Gembeck, M., (2015). Coping across the Lifespan. Em James D. Wright, International Encyclopedia of the Social \& Behavioral Sciences (pp. 887-894), 2nd edition, Vol 4. Oxford: Elsevier.

Tolinski, C. (2015). Examining academic engagement among elementary students: The role of parent-child and teacher-student relationships. Wayne State University.

Zimmer-Gembeck, M. J., \& Skinner, E. A. (2016). The development of coping: implications for psychopathology and resilience. In D. Cicchetti (Ed.), Developmental Psychopathology, (pp. 1-61). New York: Wiley. doi: 10.1002/9781119125556.devpsy410

Zimmer-Gembeck, M. J., Petegem, S. V., \& Skinner, E. A. (2016). Emotion, controllability and orientation towards stress as correlates of children's coping with interpersonal stress. Motivation and Emotion, 40(1), 178-191. doi: 10.1007/s11031-015-9520-z

recebido em outubro de 2017 aprovado em janeiro de 2019

\section{Sobre as autoras}

Schwanny Roberta Costa Rambalducci Mofati Vicente é psicóloga pela Faculdade Católica Salesiana do Espírito Santo; mestre e doutora em Psicologia pela Universidade Federal do Espírito Santo (UFES). Atualmente atua como psicóloga clínica.

Fabiana Pinheiro Ramos é psicóloga (UFES), com mestrado e doutorado em Psicologia (Programa de Pós-graduação em Psicologia da UFES). Atualmente é professora adjunta do Departamento de Psicologia da UFES e membro dos grupos de pesquisa "Processos Psicológicos e Saúde" e "Análise do Comportamento: implicações clínicas e educacionais" do diretório dos grupos de pesquisa do CNPq.

Kely Maria Pereira de Paula é psicóloga (UFRJ), doutora em Psicologia pela Universidade Federal do Espírito Santo (UFES), docente do Departamento de Psicologia Social e do Desenvolvimento e do Programa de Pós-graduação em Psicologia da UFES; coordena o Laboratório de Pesquisa em Psicologia Pediátrica - LAPEPP (PPGP/UFES). 